\title{
Availability Analysis of Polytube Industry When Two Sub-System Are Simultaneous Fail
}

\author{
Shakuntla $^{a *}$, Sanjay Kajal ${ }^{b}$, A. K. Lal ${ }^{a}$, S. S. Bhatia ${ }^{a}$ and Jai Singh ${ }^{c}$ \\ ${ }^{a}$ School of Mathematics and Computer Application, T.I.E.T. Patiala, Punjab, India, ${ }^{b}$ N.I.T Kurukshetra, India \\ and ${ }^{c}$ Punjab College of Engineering, Lalru, Punjab, India
}

\begin{abstract}
The paper discusses the availability of a pipe manufacturing industry using supplementary variable technique. The plant is divided into many sections like mixture, extruder, die and cutter. The failure rates of the sub-systems are constant and the repair rates are general and variable. The mathematical equations are derived using Chapman-Kolmogorov equation. The problem is formulated using the supplementary variable technique and the solution is carried out using the Lagrange's partial differential. Long run availability of the system is also calculated for the various cases using constant transition rates.
\end{abstract}

Key Words: MATLAB, Availability, Chapman-Kolmogorov equation, Lagrange's partial differential.

\section{Introduction}

The goal of maximum production and long run availability under the given operative condition can be achieved by making the system failure free as far as possible by proper maintenance planning and control. Reliability analysis helps us to obtain the necessary information about the control of various parameters. The Polytube industry involves many processes i.e. mixture, extruder, die and cutter. The die and cutter machine can also work in reduced state. The process starts from the mixture section in which pipe mixture is prepared with the help of $\mathrm{PVC}$ rising, $\mathrm{CaCO}_{3}$, citric acid and wax which is heated up to $130^{\circ} \mathrm{C}$. The heated material is then cooled up to $100^{\circ} \mathrm{C}$ and transported to the Extruder by conveyors. With the help of Die and Extruder, pipe is prepared .After smoothing the pipe, sorting process take place. In this process, the pipe carried to Cutter is cut into different sizes as per the need and requirement.

The mechanical systems have attracted the attention of several researchers in the area of reliability theory. Dhillon and Natesan (1983) discussed the power system in fluctuating environment.Kumar et. al. (1988) discussed about feeding systems in the sugar industry and paper industry. Kumar and Singh (1989) analyzed the Availability of a washing system of paper industry. Singh and Pandey $(1992,1990)$ discussed the reliability and availability of Fertilizer and Sugar industry.Dyal and Singh (1992) studied reliability analysis of a system in a fluctuating environment. Singh and Mahajan (1999) examined the reliability and long run availability of a
Utensils Manufacturing Plant using Laplace transforms. Gupta et. al. (2005) studied the behavior of Cement manufacturing plant. Kiureghian and Ditlevson (2007) analyzed the availability, reliability and downtime of system with repairable components. Kumar et. al. (1991) discussed the behaviour analysis of Urea decomposition in the Fertilizer industry under general repair policy. Kumar et.al. analyzed (1990) the designed and cost of a refining system in the sugar industry using supplementary variable technique.

\section{System Description}

The Polytube industry consists of four subsystems, namely

\section{Sub-system A (Mixture)}

It mixes raw material such as PVC , calcium carbonate, wax and other chemicals in appropriate proportion for manufacturing pipe .It consists of a heater by which the raw material is heated up to $130^{\circ} \mathrm{C}$ and transported to the extruder by conveyors. It consists of blades and a motor whose failure cause complete failure of the system.

\section{Sub-system B (Extruder)}

Raw material obtained from mixer is heated in this section. It consists of a heater to heat the raw material at different temperatures. The quality of the product depends upon heating process. Its failure causes the complete failure of the system.

\footnotetext{
*Corresponding author. E-mail: shakus25@gmail.com
} 


\section{Sub-system C (Die)}

It is used to make different sizes of pipe .Minor failure of the sub-system reduced the capacity of the system and hence loss in production. Major failure results in complete failure of the system.

\section{Sub-system D (Cutter)}

This sub-system has two units arranged in series. First unit is blade which cut the pipe and the second unit is motor which cut the pipe in different size. Failure of blade reduces the capacity of the system while the failure of motor causes the complete failure of the system.

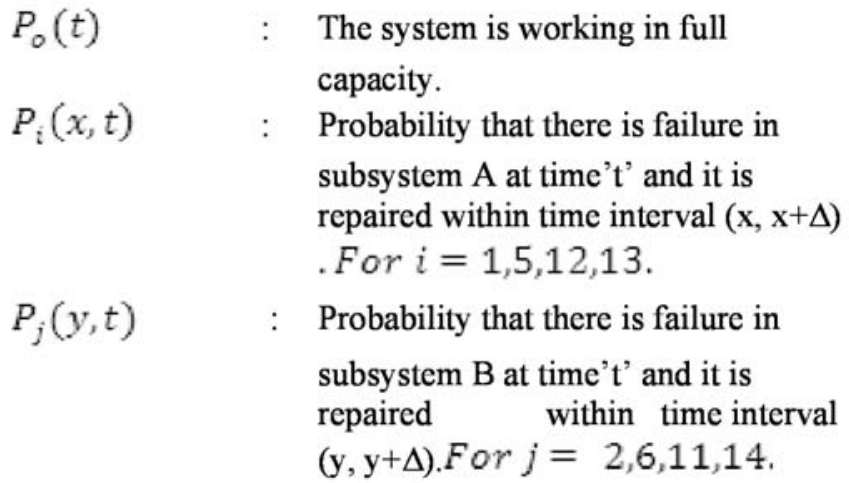

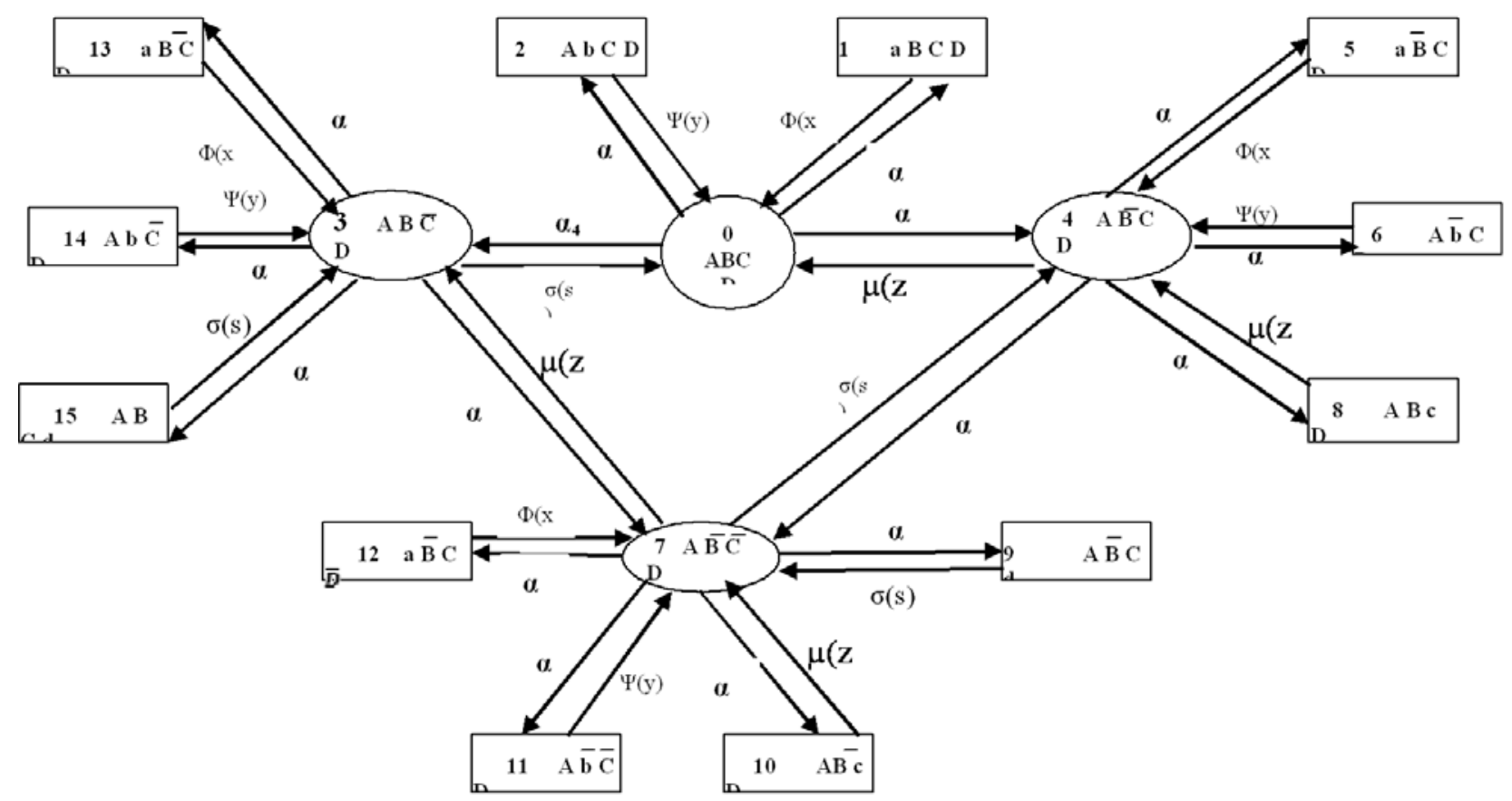

Fig. 1: Transition Diagram of Polytube Industry

\section{Assumptions}

$\mathrm{A}, \mathrm{B}, \mathrm{C}, \mathrm{D} \quad$ : Indicates that the sub-system is working in full capacity.

$\bar{C}, \bar{D}$

a, b, c, d : Indicate the failed state of the subsystem.

$\alpha_{\mathrm{i}}$ Failure rate of the sub-system $A, B, C, D$.

$\phi(\mathrm{x}), \psi(\mathrm{y}) \quad$ : $\quad$ General repair rates of $A, B, C, D$ $\mu(\mathrm{z}), \sigma(\mathrm{s})$ respectively.
$P_{3}(s, t) \quad: \quad$ Probability that there is failure in subsystem $D$ at time ' $\mathrm{t}$ ' and system remains in reduced state till it is repaired within time interval $(s, s+4)$.

$P_{4}(z, t) \quad: \quad$ Probability that there is failure in subsystem $C$ at time ' $t$ ' and system remains in reduced state till it is repaired within time interval $(z, z+\Delta)$. 
$P_{7}(s, z, t) \quad:$ Probability that there is failure in subsystem $C$ and $D$ at time 't' and system remains in reduced state till it is repaired within time interval $(z, z+\Delta)$ and $(s, s+\Delta)$ respectively.

$P_{k}(z, t) \quad:$ Probability that there is failure in subsystem $C$ at time ' $t$ ' and it is repaired within time interval $(z, z+\Delta)$. For $k=8,10$.

$P_{l}(z, t) \quad$ : Probability that there is failure in subsystem $D$ at time ' $\mathrm{t}$ ' and it is repaired within time interval $(s, s+\Delta)$. For $l=9,15$.

The assurnptions used in developing the performance model are as follows

1. Failure rates are constant over time and statistically independent.

2. A repaired unit as good as new, performance wise, for a specified duration.

3. Sufficient repair facilities are provided.

4. Service includes repair and/or replacement.

5. System may work at reduced capacity.

6. There are simultaneous failures

\section{Mathematical Formulation}

$\left[\frac{d}{d t}+S_{0}(t)\right] P_{0}(t)=M_{0}(t)$

$\left[\frac{\partial}{\partial t}+\frac{\partial}{\partial s}+M_{1}(s, t)\right] P_{3}(s, t)=M_{1}(s, t)$

$\left[\frac{\partial}{\partial t}+\frac{\partial}{\partial z}+S_{2}(z)\right] P_{4}(z, t)=M_{2}(z, t)$

$\left[\frac{\partial}{\partial t}+\frac{\partial}{\partial s}+\frac{\partial}{\partial z}+s_{3}(s, z)\right] P_{7}(s, z, t)=M_{3}(s, z, t)$

$\left[\frac{\partial}{\partial t}+\frac{\partial}{\partial x}+\phi(x)\right] P_{1}(x, t)=0$

$\left[\frac{\partial}{\partial t}+\frac{\partial}{\partial y}+\psi(y)\right] P_{2}(y, t)=0$

$\left[\frac{\partial}{\partial t}+\frac{\partial}{\partial x}+\phi(x)\right] P_{5}(x, t)=0$

$\left[\frac{\partial}{\partial t}+\frac{\partial}{\partial y}+\psi(y)\right] P_{6}(y, t)=0$

$\left[\frac{\partial}{\partial t}+\frac{\partial}{\partial z}+\mu(z)\right] P_{8}(z, t)=0$

$\left[\frac{\partial}{\partial t}+\frac{\partial}{\partial s}+\sigma(s)\right] P_{g}(s, t)=0$

$\left[\frac{\partial}{\partial t}+\frac{\partial}{\partial z}+\mu(z)\right] P_{10}(z, t)=0$ $\left[\frac{\partial}{\partial t}+\frac{\partial}{\partial y}+\psi(y)\right] P_{11}(y, t)=0$

$\left[\frac{\partial}{\partial t}+\frac{\partial}{\partial x}+\phi(x)\right] P_{12}(x, t)=0$

$\left[\frac{\partial}{\partial t}+\frac{\partial}{\partial x}+\phi(x)\right] P_{13}(x, t)=0$

$\left[\frac{\partial}{\partial t}+\frac{\partial}{\partial y}+\psi(y)\right] P_{14}(y, t)=0$

$\left[\frac{\partial}{\partial t}+\frac{\partial}{\partial s}+\sigma(s)\right] P_{15}(s, t)=0$

\section{Initial Conditions}

$P_{0}(0)=1$ otherwise 0

$P_{i}(x, 0)=0$ For $i=1,5,12,13$

$P_{j}(y, 0)=0$ For $j=2,6,11,14$

$P_{k}(z, 0)=0$ For $k=4,7,8,10$

$P_{l}(s, 0)=0$ For $l=3,7,9,15$

\section{Boundary Conditions}

$$
\begin{aligned}
& P_{1}(0, t)=\alpha_{1} P_{0}(t) \\
& P_{2}(0, t)=\alpha_{2} P_{0}(t) \\
& P_{3}(0, t)=\alpha_{4} P_{0}(t) \\
& P_{4}(0, t)=\alpha_{3} P_{0}(t) \\
& P_{5}(0, t)=\int \alpha_{1} P_{4}(z, t) d z \\
& P_{6}(0, t)=\int \alpha_{2} P_{4}(z, t) d z \\
& P_{7}(0,0, t)=\int \alpha_{3} P_{3}(s, t) d s+\int \alpha_{4} P_{4}(z, t) d z
\end{aligned}
$$

$P_{8}(0, t)=\int \alpha_{3} P_{4}(z, t) d z d z$

$P_{9}(0, t)=\int \alpha_{4} P_{7}(s, z, t) d s d z$

$P_{10}(0, t)=\int \alpha_{3} P_{7}(s, z, t) \mathrm{d} s \mathrm{~d} z$

$P_{11}(0, t)=\int \alpha_{2} P_{7}(s, z, t) \mathrm{d} s \mathrm{~d} z$

$P_{12}(0, t)=\int \alpha_{1} P_{7}(s, z, t) d s d z$

$P_{13}(0, t)=\int \alpha_{1} P_{3}(s, t)$

$P_{14}(0, t)=\int \alpha_{2} P_{3}(s, t) d s$

$P_{15}(0, t)=\int \alpha_{4} P_{3}(s, t) d s$

For further solving equation (1-16) we have made the following notations

$S_{0}(t)=\sum_{i=1}^{4} \alpha_{i}$

$M_{0}(t)=\int P_{1}(x, t) \phi(x) d x+\int P_{2}(y, t) \psi(y) d y+$

$\int P_{3}(s, t) \sigma(s) d s+\int P_{4}(z, t) \mu(z) d z$ 


$$
\begin{array}{ll}
S_{3}(s, z)=\sum_{i=1}^{4} \alpha_{i}+\sigma(s)+\mu(z) & P_{3}(s, t)=e^{-\int s_{1}(s) d s}\left[\alpha_{4} P_{0}(t-s)+\right. \\
S_{0}(t)=\sum_{i=1}^{4} \alpha_{i} & \left.\int M_{1}(s, t) e^{\int s_{1}(s) d s} d s\right] \\
& P_{3}(s, t)=e^{-\int s_{1}(s) d s}\left[\alpha_{4} P_{0}(t-s)+\right. \\
P_{1}(x, t) \phi(x) d x+\int P_{2}(y, t) \psi(y) d y+\int P_{3} & \left.\int M_{1}(s, t) e^{\int s_{1}(s) d s} d s\right] \\
S_{3}(s, z)=\sum_{i=1}^{4} \alpha_{i}+\sigma(s)+\mu(z) & P_{0}(t)=e^{-\int s_{0}(t) d t}\left[1+\int M_{0}(t) e^{\int s_{0}(t) d t} d t\right]
\end{array}
$$$$
S_{0}(t)=\sum_{i=1}^{4} \alpha_{i}
$$$$
{ }_{1}(x, t) \phi(x) d x+\int P_{2}(y, t) \psi(y) d y+\int P_{3}(
$$$$
S_{3}(s, z)=\sum_{i=1}^{4} \alpha_{i}+\sigma(s)+\mu(z)
$$$$
S_{0}(t)=\sum_{i=1}^{4} \alpha_{i}
$$

${ }_{1}(x, t) \phi(x) d x+\int P_{2}(y, t) \psi(y) d y+\int P_{3}($

The system of partial differential equations (1-16) together with the initial conditions (17-21) and boundary conditions (22-36) are solved by Lagrange's method we get

$P_{1}(x, t)=\alpha_{1} P_{0}(t-x) e^{-\int \phi(x) d x}$

$P_{2}(y, t)=\alpha_{2} P_{0}(t-y) e^{-\int \psi(y) d y}$

$P_{5}(x, t)=e^{-\int \phi(x) d x} \int \alpha_{1} P_{4}(z, t-x) d z$

$P_{6}(y, t)=e^{-\int \psi(y) d y} \int \alpha_{2} P_{4}(z, t-y) d z$

$P_{8}(z, t)=e^{-\int \mu(z) d z} \int \alpha_{3} P_{4}(z, t-z) d z$

$P_{9}(s, t)=e^{-\int \sigma(s) d s} \int \alpha_{4} P_{7}(s, z, t-s) d s d z$

$P_{10}(z, t)=e^{-\int \mu(z) d z} \int \alpha_{3} P_{7}(s, z, t-z) d s d z$ (43)

$P_{11}(y, t)=e^{-\int \psi(y) d y} \int \alpha_{2} P_{7(3)}(s, z, t-y) d s d z$

$P_{12}(x, t)=e^{-\int \phi(x) d x} \int \alpha_{1} P_{7(3)}(s, z, t-x) d s d z$

$P_{13}(x, t)=e^{-\int \phi(x) d x} \int \alpha_{1} P_{3}(s, t-x) d s$

$P_{14}(y, t)=e^{-\int \psi(y) d y} \int \alpha_{2} P_{3}(s, t-y) d s$

$P_{15}(s, t)=e^{-\int \sigma(s) d s} \int \alpha_{4} P_{3}(s, t-s) d s$

$P_{7}(s, z, t)=e^{-\int S_{z}(s, z) d s}$

$\left[\begin{array}{c}\int \alpha_{3} P_{3}(s, t-z) d s+\int \alpha_{4} P_{4}(z, t-s) d z \\ +\int M_{3}(s, z, t) e^{\int s_{3}(s, z) d s} d s\end{array}\right]$
It is evident that all probabilities are obtained in terms of $P_{0}(t)$ which is given by (1)

\section{The time dependent availability $A(t)$ is}

$\left[\left(\alpha_{1}+\alpha_{2}+\alpha_{3}+\alpha_{4}+\sigma+\mu\right] P_{7}=\alpha_{3} P_{3}+\alpha_{4} P_{4}\right.$

$+\sigma P_{9}+\mu P_{10}+\psi P_{11}+\phi P_{12}$

In the process industry, we require long run availability of the system, which is obtained by putting $\frac{d}{d t}=0, \frac{\partial}{\partial t}=0$ and taking probabilities independent of " $\mathrm{t}$ "

For steady state availability transition rates are taken to be constant.

$P_{3}(s, t)=e^{-\int s_{1}(s) d s}\left[\alpha_{4} P_{0}(t-s)+\int M_{1}(s, t) e^{\int s_{1}}\right.$$$
\left[\left(\alpha_{1}+\alpha_{2}+\alpha_{3}+\alpha_{4}+\sigma+\mu\right] P_{7}=\alpha_{3} P_{3}+\alpha_{4} F\right.
$$

${ }_{4}+\sigma P_{9}+\mu P_{10}+\psi P_{11}+\phi P_{12}$

$\left[\left(\alpha_{1}+\alpha_{2}+\alpha_{3}+\alpha_{4}+\sigma+\mu\right] P_{7=} \alpha_{3} P_{3}+\alpha_{4}\right.$

$P_{4}+\sigma P_{9}+\mu P_{10}+\psi P_{11}+\phi P_{12}$

$\left[\left(\alpha_{1}+\alpha_{2}+\alpha_{3}+\alpha_{4}+\sigma+\mu\right] P_{7=} \alpha_{3} P_{3}+\right.$

$\alpha_{4} P_{4}+\sigma P_{9}+\mu P_{10}+\psi P_{11}+\phi P_{12}$

$\phi \mathrm{P}_{1}=\alpha_{1} \mathrm{P}_{0}$

$\psi P_{2}=\alpha_{2} P_{0}$

$\phi P_{5}=\alpha_{1} P_{4}$

$\psi P_{6}=\alpha_{2} P_{4}$

$\mu P_{8}=\alpha_{3} P_{4}$

$\sigma P_{9}=\alpha_{4} P_{7}$

$\mu P_{10}=\alpha_{3} P_{7}$

$\psi P_{11}=\alpha_{2} P_{7}$

$\phi P_{12}=\alpha_{1} P_{7}$ 
$\phi P_{13}=\alpha_{1} P_{3}$

$\psi P_{14}=\alpha_{2} P_{3}$

$\sigma P_{15}=\alpha_{4} P_{3}$

On solving equations (54-69) recursively, we get

$P_{7}=\frac{\alpha_{3}}{s_{1}} P_{3}+\frac{\alpha_{4}}{s_{1}} P_{4}$

$P_{4}=\frac{\alpha_{3}}{s_{2}} P_{0}+\frac{\sigma \alpha_{3}}{s_{1} s_{2}} P_{3}$

$P_{3}=\left(\frac{\alpha_{4}}{s_{3}}+\frac{\alpha_{3} \mu \alpha_{4}}{s_{1} s_{2} s_{3}}\right) P_{0}$

Now using normalizing conditions

$\sum_{i=0}^{15} P_{i}=1$

$P_{0}=\left[1+\frac{\alpha_{1}}{\phi}+\frac{\alpha_{2}}{\psi}+\left(1+\frac{\alpha_{1}}{\phi}+\frac{\alpha_{2}}{\psi}+\frac{\alpha_{4}}{\sigma}\right) M_{1}+\right.$

$\left(1+\frac{\alpha_{1}}{\phi}+\frac{\alpha_{2}}{\psi}+\frac{\alpha_{3}}{\mu}\right) M_{2}+\left(1+\frac{\alpha_{1}}{\phi}+\frac{\alpha_{2}}{\psi}+\frac{\alpha_{4}}{\sigma}+\right.$

$\left.\left.\frac{\alpha_{3}}{\mu}\right) M_{3}\right]^{-1}$

Once $P_{0}$ is determined the probabilities of other states $P_{1}, P_{2}, P_{3}, . ., P_{15}$ can also be obtained. Finally, we can calculated the availability of the system

$$
\begin{aligned}
& A_{v}=\left[1+M_{1}+M_{2}+M_{3}\right] P_{0} \\
& s_{1}=\sigma+\mu \\
& s_{2}=\alpha_{4}+\mu-\frac{\sigma \alpha_{4}}{s_{1}} \\
& s_{3}=\alpha_{3}+\sigma-\frac{\mu \alpha_{3}}{s_{1}}-\frac{\sigma \mu \alpha_{4} \alpha_{3}}{s_{1} s_{2} s_{1}} \\
& \mathrm{M}_{1}=\left(\frac{\alpha_{4}}{s_{3}}+\frac{\alpha_{3} \mu \alpha_{4}}{s_{2} s_{2} s_{3}}\right) \\
& M_{2}=\frac{\alpha_{3}}{s_{2}}+\frac{\sigma \alpha_{3}}{s_{1} s_{2}} M_{1} \\
& M_{3}=\frac{\alpha_{3}}{s_{1}} M_{1}+\frac{\alpha_{4}}{s_{1}} M_{2}
\end{aligned}
$$

\section{Performance Analysis and Discussion}

Tables 1, 2, 3 and 4 represent the availabilities for various subsystems of a Polytube extrusion. These tables reveal the various availability levels for different combinations of failure events and repair priorities. On the basis of possible combination $S_{3}(s, z)=\sum_{i}^{4}$ i.e. optimal maintenance strategy, we may select the maximum value of availability for each subsystem. So, the optimal values of failure and repair rates may be selected accordingly for each subsystem of the Polytube extrusions Table 1 shows that as the repair rates of the mixture increases, the availability of Polytube Extrusion system increases drastically whereas the Table II shows that with the increase in repair rates of the extruder, the availability of the system increase appreciably.

Table I: Effect of failure and repair rate of the sub-system Mixture (A) on Availability

\begin{tabular}{c|c|c|c|c|l}
\hline$\alpha_{1}$ & 0.0057 & 0.0059 & 0.0061 & 0.0063 & Constant values \\
$\square \bigvee$ & & & & & \\
\hline 0.5 & 0.9853 & 0.9849 & 0.9845 & 0.9841 & $\alpha_{2}=0.007, \psi=2$ \\
0.7 & 0.9884 & 0.9882 & 0.9879 & 0.9876 & $\alpha_{3}=0.01, \mu=0.33$ \\
0.9 & $0 . .9902$ & 0.9900 & 0.9898 & 0.9896 & $\alpha_{4}=0.015, \sigma=0.02$ \\
1.1 & 0.9913 & 0.9912 & 0.9910 & 0.9908 & \\
\hline
\end{tabular}

Table II: Effect of failure and repair rate of the sub-system Extruder (B) on Availability

\begin{tabular}{|c|c|c|c|c|c|}
\hline${ }_{\mu}^{\alpha_{3}}$ & 0.01 & 0.02 & 0.03 & 0.04 & Constant values \\
\hline 0.33 & \begin{tabular}{|l|}
0.9853 \\
\end{tabular} & 0.9853 & 0.9853 & 0.9853 & $\alpha_{1}=0.0057, \square=0.5$ \\
\hline 0.53 & 0.9853 & 0.9853 & 0.9853 & 0.9853 & $\alpha_{2}=0.07, \psi=2$ \\
\hline 0.73 & $0 . .9854$ & 0.9854 & 0.9854 & 0.9855 & $\alpha_{4}=0.015, \sigma=2$ \\
\hline .93 & 0.9854 & 0.9854 & 0.9854 & 0.9855 & \\
\hline
\end{tabular}

\begin{tabular}{l|c|c|c|c|l}
\hline$\alpha_{2}$ & 0.007 & 0.009 & 0.011 & 0.013 & Constant values \\
\hline 2 & & & & & \\
4 & 0.9853 & 0.9843 & 0.9832 & 0.9823 & $\alpha_{1}=0.0057, \square=0.5$ \\
6 & 0.9869 & 0.9865 & 0.9860 & 0.9855 & $\alpha_{3}=0.01, \mu=0.33$ \\
8 & 0.9878 & 0.9872 & 0.9869 & 0.9865 & $\alpha_{4}=0.015, \sigma=2$ \\
\hline
\end{tabular}

Table III and IV shows that there is a almost negligible change in the availability of the Polytube Extrusion system

\begin{tabular}{|c|c|c|c|c|c|}
\hline$\alpha_{4}$ & 0.015 & 0.030 & 0.45 & 0.060 & Constant values \\
\hline 2 & 0.9853 & 0.9853 & 0.9853 & 0.9853 & $\alpha_{1}=0.0057, \square=0.5$ \\
\hline 4 & 0.9853 & 0.9853 & 0.9853 & 0.9852 & $\alpha_{2}=0.07, \psi=0.04$ \\
\hline 6 & $0 . .9853$ & 0.9852 & 0.9852 & 0.9852 & $\alpha_{3}=0.33, \mu=0.02$ \\
\hline 8 & 0.9854 & 0.9853 & 0.9853 & 0.9852 & \\
\hline
\end{tabular}
with the increase the repair rate of Die and Cutter subsystem.

Table III: Effect of failure and repair rate of the sub-system Die (C) on Availability

Table IV: Effect of failure and repair rate of the sub-system Cutter (D) on Availability 


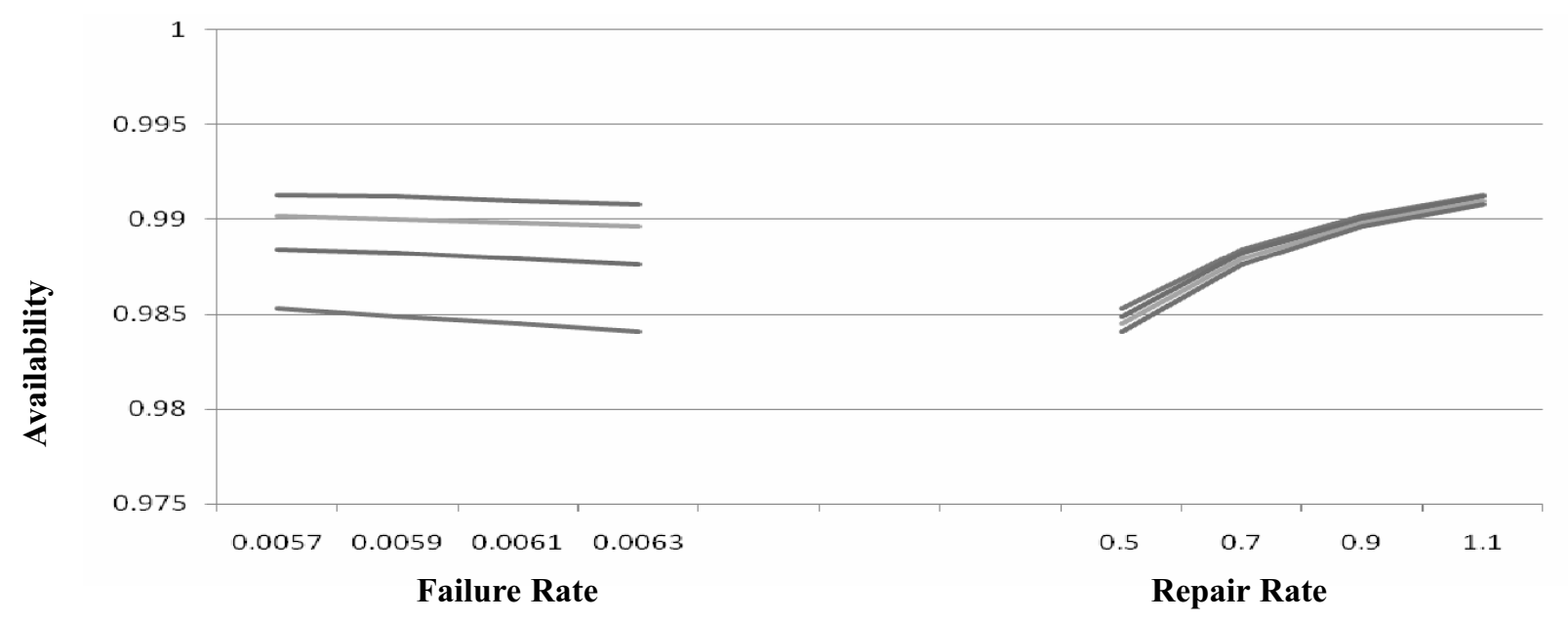

Fig. 2: Effect of failure and repair rate of Mixture on availability.

Therefore, the Mixture subsystem is the most critical as far as maintenance is concerned and should be taken on topmost priority and results are also shown graphically by figure 2 .

Under the available facilities the concern management may minimize the failure time of each sub-system adopting the following measures

i) Getting the information of failure of each equipment at the earliest moment.

ii) Starting the repair work at the earliest moment.

iii) Providing trained workers.

iv) Providing the special tools required.

v) Making available the spare parts and special parts

\section{Acknowledgement}

Author is thankful to the learned referee for his valuable suggestions and would like to thank Sh. Sudarshan Aggarwal, Managing Director, Polytube Industry, Kurukshetra for providing necessary data's of this industry.

\section{References}

Dhillon B. S. and Natesan J. (1983). Stochastic analysis of a system in a fluctuating environment, Micro electron Reliability, 23: 867-881.

Dayal B. and Singh J. (1992). Reliability analysis of a system in a fluctuating environment, Micro electron Reliability, 32: 601-3.

Gupta P., Lal A. K., Sharma R. K. and Singh J. (2005). Behavioral Study of the Cement manufacturing Plant A Numerical Approach, Journal of Mathematics and Systems Sciences, 1(1): 50-69.

Kumar D., Singh J. and Singh I. P. (1988). Reliability analysis of the feeding system in the paper industry, Microelectron Reliability, 28: 213-215.
Kumar D., Singh J. and Singh I. P. (1988). Availability of the feeding system in the sugar industry, Microelectron Reliability, 28: 867-871.

Kumar D. and Singh J. (1989). Availability of a Washing System in the Paper Industry, Microelectron. Reliability, 29: 775-778.

Kumar D., Singh J., and Pandey P. C. (1992). Availability of the Crystallization System in the Sugar Industry under Common - Cause Failure, IEEE Transactions on Reliability, 41 (1): 85-91.

Kiureghian A. D. and Ditlevson O. D. (2007), Availability, Reliability \& downtime of system with repairable components, Reliability Engineering and System Safety, 92 (2): 66-72.

Singh J., Pandey P. C. and Kumar D. (1990). Designing for Reliable Operation of Urea Synthesis in the Fertilizer Industry, Microelectron. Reliability 30: 1021-1024.

Singh J. and Mahajan P. (1999). Reliability of Utensils Manufacturing Plant - A Case Study, Opsearch, 36 (3): 260-269.

Kumar D., Pandey P. C. and Singh J. (1991). Behaviour analysis of Urea decomposition in the Fertilizer industry under general repair policy, Micro electron. Reliability, 31(5): 851-854.

Kumar D., Pandey P. C. and Singh J. (1990). Design and cost analysis of a refining system in a Sugar industry, Micro electron. Reliability, 30(6): 1025-1028.

Received : June 06, 2010;

Accepted : February 17, 2011 